\title{
ISODIAMETRIC AND ISOPERIMETRIC INEQUALITIES FOR COMPLEXES AND GROUPS
}

\author{
P. PAPASOGLU
}

\begin{abstract}
It is shown that D. Cohen's inequality bounding the isoperimetric function of a group by the double exponential of its isodiametric function is valid in the more general context of locally finite simply connected complexes. It is shown that in this context this bound is 'best possible'. Also studied are seconddimensional isoperimetric functions for groups and complexes. It is shown that the second-dimensional isoperimetric function of a group is bounded by a recursive function. By a similar argument it is shown that the area distortion of a finitely presented subgroup of a finitely presented group is recursive. Cohen's inequality is extended to second-dimensional isoperimetric and isodiametric functions of 2-connected simplicial complexes.
\end{abstract}

\section{Introduction}

D. Cohen showed in [3] that the isoperimetric function (also called the Dehn function) of a group is bounded by the double exponential of its isodiametric function. It is an open question as to whether this bound is optimal. Gersten in [6] gave a geometric proof of Cohen's inequality. We show in this paper that Cohen's inequality is valid in the more general context of simply connected simplicial complexes. Our proof is similar to that of Gersten. We construct an example showing that the double exponential bound is optimal for locally finite simply connected simplicial complexes. Gromov remarked in [9] that if one could show that the filling length of a disk was bounded by its diameter, then one could improve Cohen's double exponential bound to a simple exponential (see [9, Section 5C] for definitions and a precise statement). Frankel and Katz in [5], however, showed that this is impossible. Their counterexample however does not show that Cohen's inequality is optimal even in the context of locally finite simply connected simplicial complexes. Indeed the disks they construct are non-singular and for such disks it is easy to see that the area is bounded by the exponential of their diameter.

We show that D. Cohen's bound extends to second-dimensional isoperimetric functions and that it is optimal in this context as well. If a group is of type $\mathrm{FP}_{3}$ then one can define its second Dehn function. We show that (in contrast with usual Dehn functions) the second Dehn function of a group is always bounded by a recursive function. By a similar argument we show that area distortion of subgroups is also always recursive. This contrasts with the fact that length distortion of subgroups can be non-recursive. Area distortion has been studied by Gersten [7] who showed that the area distortion of subgroups of a group with solvable word problem is recursive. Two-dimensional isoperimetric inequalities have been studied by several people (see $[1,2,4,9,10,16])$. A number of questions answered for usual Dehn functions remain open for second Dehn functions. We mention some of them.

Received 25 February 1999; revised 6 July 1999.

2000 Mathematics Subject Classification 20F32, 20F10.

J. London Math. Soc. (2) 62 (2000) 97-106 
Question 1.1. Are there groups whose second Dehn function grows faster than $\exp (n)$ ?

We remark that Otto and Madlener [12] have shown that for every recursive function $f$ there is a finitely presented group $G$ which contains a subgroup $H$ such that the area distortion of $H$ in $G$ grows faster than $f$. If one could construct such a group $G$ of type $\mathrm{FP}_{3}$ then the group $G \star_{H} G$ would have a second Dehn function growing faster than $f$. In fact it would be sufficient for this construction to embed $G$ in a group of type $\mathrm{FP}_{3}$, which suggests the following question.

Question 1.2. Can one embed any finitely presented group into a group of type $\mathrm{FP}_{3}$ ?

This is a 'higher dimensional' analogue of Higmann's theorem stating that every recursively presented group can be embedded into a finitely presented group.

The filling radius function, denoted by FR, of a group $G$ (or a simply connected complex) is defined as follows. The filling radius of a simple closed path $c$ in the 1 -skeleton of a Cayley complex of $G$ is the smallest $n$ such that $c$ is contractible in its $n$-neighbourhood. We define now

$$
\operatorname{FR}(n)=\max \{\text { filling radius of } c \text { where length }(c) \leqslant n\} \text {. }
$$

It is shown in [8, Section 6] (see also [13]) that if $\mathrm{FR}(n)$ is sublinear then it is bounded by $M \log (n)(M>0)$. In fact if $\mathrm{FR}(n)$ is sublinear for a group $G$ then $G$ is hyperbolic. Moreover it is not hard to see that if $\operatorname{FR}(n)$ is sublogarithmic (that is, for all $M>0$, $\mathrm{FR}(n) \leqslant M \log (n)$ for almost all $n)$ then it is actually bounded, which implies that $G$ is virtually free. Clearly the filling radius function can be generalized to higher dimensions. Let us denote by $\mathrm{FR}_{2}$ the second-dimensional filling radius function of a group. We have then the following question.

Question 1.3. Let $G$ be a group of type $\mathrm{FP}_{3}$ whose (usual) Dehn function is quadratic. Suppose that the second-dimensional filling radius function of $G, \operatorname{FR}_{2}(n)$, is sublogarithmic. Is it then bounded? Suppose that $\mathrm{FR}_{2}(n)$ is sublinear; is it then bounded by $c \log (n)$ ?

Of course this question makes sense for 2-connected complexes in general.

\section{Isodiametric inequalities}

Let $X$ be a simply connected locally finite simplicial complex. Let $f: S^{1} \longrightarrow X$ be a simplicial map and let $\bar{f}: D \longrightarrow X$ be a simplicial map from the 2-disc to $X$ such that $\left.\bar{f}\right|_{\partial(D)}=f$. Let $v$ be a vertex of $S^{1}$. We define

$$
\operatorname{diam}_{v}(\bar{f})=\max _{u \in D^{(0)}} d_{D^{(1)}}(v, u)
$$

where $d_{D^{(1)}}$ is the singular metric induced on $D^{(1)}$ by $\bar{f}$. In other words if $v, u \in D^{(1)}$ we define

$$
d_{D^{(1)}}(v, u)=\min \{\text { length }(f(p)): p \text { path joining } v \text { to } u\}
$$

where length $(f(p))$ is the reduced length of $f(p)$ as a path in $X$. We remark that if $X$ is the Cayley complex of a group and $D$ is a Van Kampen diagram, then $d_{D^{(1)}}$ is the word metric on $D^{(1)}$. We define

$$
\operatorname{diam}_{v}(f)=\min \left\{\operatorname{diam} \bar{f}:\left.\bar{f}\right|_{\partial(D)}=f\right\} .
$$


D. Cohen (see [3]) showed that there are constants $a, b$ such that

$$
\operatorname{area}(f) \leqslant \operatorname{length}(f) a^{b^{\mathrm{diam}(f)}} .
$$

To be more precise $\mathrm{D}$. Cohen showed the inequality above in the case where $X$ is the Cayley complex of a finitely presented group. S. Gersten (see [6]) has given a geometric proof of this result. It is not hard to see that Gersten's proof applies generally when $X$ is a locally finite complex.

Proposition 1.1. Let $X$ be a locally finite simply connected simplicial complex. There is a constant $k$ such that for every path $f: S^{1} \longrightarrow X$ in $X$ we have

$$
\text { area }(f) \leqslant \text { length }(f) 3^{k^{\text {diam }(f)}} \text {. }
$$

Proof. Let $v$ be a vertex of $f$ lying on $f\left(S^{1}\right)$. We consider the set $S$ of all loops lying in $X^{(1)}$ of the form $p u p^{-1}$, where $p$ is a path in $X^{(1)}$ of length less than or equal to $\operatorname{diam}(f)$ and $u$ is a path going around a 2 -simplex of $X$. We use the set $S$ to construct a labelled 2-complex $Y$ as follows. We associate to a path pup $^{-1}$ in $S$ a simplicial segment consisting of as many edges as $p$ joined with a 2-simplex. We map the 1skeleton of this complex to the path pup $^{-1}$ in the obvious way and we map the 2simplex to the 2-simplex in $X$ having $u$ as boundary. We label now the simplices of this complex by their image in $X$ (that is, the 'alphabet' used in this labelling is the simplices of $X$ ). To form $Y$ we identify all the complexes corresponding to paths in $S$, along the first vertex (the one labelled by $v$ ) of their corresponding segments.

It is clear that $Y$ satisfies a linear isoperimetric inequality. In fact for every closed path $q$ in $Y$ we have

$$
\operatorname{area}(q) \leqslant \frac{1}{3} \text { length }(q)
$$

so if we denote by $\delta_{Y}$ the Dehn function of $Y$ we have $\delta_{Y}(n) \leqslant n / 3$. We now perform a 'folding' operation: if $e_{1}, e_{2}$ have a vertex in common and they are labelled by the same edge of $X$ then we identify them. We create in this way a complex $Y_{1}$. If there are still edges that can be folded we continue doing so, creating successively complexes $Y_{2}, Y_{3}, \ldots, Y_{n}$. Since the number of edges of $Y$ is bounded by $k^{\mathrm{diam}(f)}$, where $k$ is greater than or equal to the cardinality of $\operatorname{star}(x)$ for all $x \in X$, we have

$$
n \leqslant k^{\mathrm{diam}(f)} .
$$

Consider now $Y_{i}, Y_{i+1}$ and let $\delta_{i}, \delta_{i+1}$ be their corresponding Dehn functions. We claim that

for all $n$.

$$
\delta_{i+1}(n) \leqslant \delta_{i}(3 n)
$$

Let $h: Y_{i} \longrightarrow Y_{i+1}$ be the folding map from $Y_{i}$ to $Y_{i+1}$. We have then $h\left(e_{1}\right)=$ $h\left(e_{2}\right)=e$ for some oriented edges $e_{1}, e_{2}$ of $Y_{i}$. Let $q$ be a closed path in $Y_{i+1}$. If $q$ can be lifted to a closed path in $Y_{i}$ by $h$ then the area of $q$ in $Y_{i+1}$ is less than or equal to the area of $q$ in $Y_{i}$. If on the other hand $q$ cannot be lifted then $q$ goes through the endpoint of $e$. We associate then a path $q^{\prime}$ to $q$ as follows. We consider $h^{-1}(q)$. If $d_{1}, d_{2}$ are successive edges of $q$ and the endpoint of $h^{-1}\left(d_{1}\right)$ is not equal to the origin of $h^{-1}\left(d_{2}\right)$ then we join them by the path $e_{1}^{-1} e_{2}$. In this way we obtain a path $q^{\prime}$ in $Y_{i}$ with

$$
\text { length }\left(q^{\prime}\right) \leqslant 3 \text { length }(q) \text {. }
$$

Now a filling disk for $q^{\prime}$ projects to a filling disk for $q$ by $h$. This clearly implies that $\delta_{i+1}(n) \leqslant \delta_{i}(3 n)$.

We see therefore inductively that if $q$ is a closed path in $Y_{n}$

$$
\operatorname{area}(q) \leqslant \delta_{Y}\left(3^{n} \text { length }(q)\right) \leqslant 3^{n} \text { length }(q) .
$$


Let $g: Y_{n} \longrightarrow X$ be the map sending each simplex in $Y_{n}$ to each label (we recall that the labels are simplices of $X$ ).

We note now that the path $f$ can be lifted by $g$ to a path $f^{\prime}$ in $Y_{n}$. A filling disk for $f^{\prime}$ projects by $g$ to a filling disk for $f$. By inequalities (1) and (2) we have

$$
\operatorname{area}(f) \leqslant \text { length }(f) 3^{k^{\mathrm{diam}(f)}} \text {. }
$$

We will show that the inequality above is the 'best possible' for locally finite simplicial complexes. More precisely we have the following theorem.

THEOREM 1.2. There are a locally finite simply connected simplicial complex $X$ and paths $f_{n}: S^{1} \longrightarrow X$ such that

(1) length $\left(f_{n}\right)=4 n$;

(2) $\operatorname{area}\left(f_{n}\right) \geqslant 2^{2^{n}}$;

(3) $\operatorname{diam}\left(f_{n}\right) \leqslant 7 n$.

Proof. It suffices to construct singular simplicial disks $D_{n}$ such that $\partial D_{n}$ satisfy conditions (1), (2), (3) of the theorem. Then we can take $X$ to be the set of these disks joined by paths.

Our basic building blocks are circular bands $b_{n}$. The bands $b_{n}$ are simplicial complexes homeomorphic to $S^{1} \times[0,1]$. They have the property that

$$
\text { length }\left(S^{1} \times\{1\}\right)=2 \text { length }\left(S^{1} \times\{0\}\right)=2 n \text {. }
$$

Moreover they satisfy area $\left(b_{n}\right)=3 n$ (see Figure 1 for $b_{4}$ ).

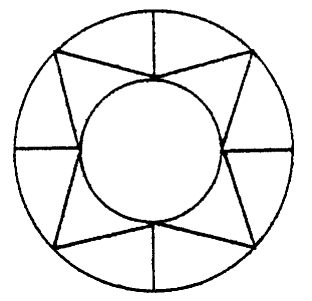

FIGURE 1. The band $b_{4}$.

It is easy to construct a simplicial disk $C_{1}$ with

$$
\text { length }\left(\partial C_{1}\right)=4 n, \quad \operatorname{area}\left(C_{1}\right)=6 \cdot\left(2^{n-1}-1\right) .
$$

Moreover each vertex of $C_{1}$ belongs to at most seven 2-simplices.

Let $c_{1}=\partial C_{1}$ (see Figure 2).

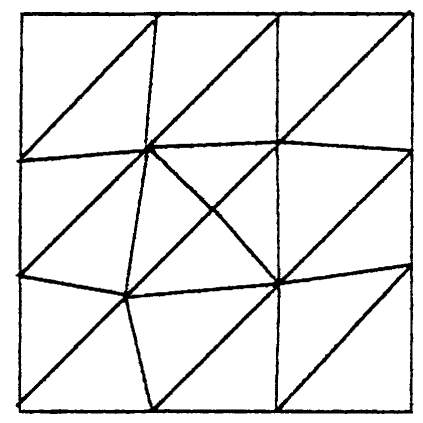

FIGURE 2. 
We will now use the band $b_{4 n}, 2^{n}$ times. Note that one component of the boundary of $b_{4 n}$ has length $8 n$ (the component $S^{1} \times\{1\}$ ). We identify this to $c_{1}$ by using a 'doubling map'. This is clearly possible since length $\left(c_{1}\right)=4 n$.

Thus this component of the boundary of $b_{4 n}$ goes around $c_{1}$ twice. If we denote by $c_{2}$ the component of the boundary of $c_{2}$ corresponding to $S^{1} \times\{0\}$ (the 'short side' of the boundary) it is then clear that

$$
\operatorname{area}\left(c_{2}\right)>2 \operatorname{area}\left(c_{1}\right) \text { and length }\left(c_{2}\right)=\text { length }\left(c_{1}\right) \text {. }
$$

We can now take one more copy of the band $b_{4 n}$ and repeat, that is, identify the 'long side' of its boundary, using a doubling map, with $c_{2}$.

Let $c_{2^{n}}$ be the path we get after we do this gluing $2^{n}$ times. Then

$$
\operatorname{area}\left(c_{2^{n}}\right)>2^{2^{n}} \text { area }\left(c_{1}\right)>2^{2^{n}} \text { and length }\left(c_{2^{n}}\right)=\text { length }\left(c_{1}\right) \text {. }
$$

On the other hand

$$
\operatorname{diam}\left(c_{2^{n}}\right)>2^{n} .
$$

We now fold some edges in order to decrease diam $\left(c_{2^{n}}\right)$. Let $s$ be a simple edge path in $C_{1}$, of length $2^{n}$ such that the initial vertex of $s, v$, lies on $c_{1}$. Let $p$ be a path of length $2^{n}$ lying in the union of the copies of the band $b_{4 n}$ and satisfying $p(0)=v$ and such that $p(i)$ is a vertex lying on the band glued in the $i$ th step of the gluing procedure described above.

We identify now $s$ with $p$ by an isometry fixing $v$. We call the resulting complex $D_{n}$. Let $f_{n}$ be the image of $c_{2^{n}}$ in $D_{n}$. It is easy to see that

$$
\operatorname{diam}\left(f_{n}\right)<7 n, \quad \text { area }\left(f_{n}\right)=\operatorname{area}\left(c_{2^{n}}\right), \quad \text { length }\left(f_{n}\right)=4 n .
$$

It is clear that each vertex in $D_{n}$ belongs to at most 142 -simplices. Clearly if we take $X$ to be the complex obtained by joining the $D_{n}, n=2,3, \ldots$, by edges then $X$ satisfies all the conditions of the theorem.

\section{Higher dimensional isoperimetric inequalities}

Let $X$ be a 2-connected locally finite simplicial complex. We identify the 2-sphere $S^{2}$ with the boundary of the standard 3-dimensional simplex and we consider simplicial maps $f: S^{2} \longrightarrow X$. By our hypothesis on $X, f$ extends to a simplicial map from the 3-ball $B^{3}$ to $X$. Using the simplicial approximation theorem (see for example [15], p. 128]) we see that there exists a simplicial refinement of the 3-ball (which we will still denote $B^{3}$ ) and a map $\bar{f}: B^{3} \longrightarrow X$ such that

$$
\left.\bar{f}\right|_{\partial B^{3}}=\left.f\right|_{\partial B^{3}}
$$

which sends each $i$-dimensional simplex of $B^{3}$ to a simplex of $X$ of dimension less than or equal to $i$. We define the volume of $\bar{f}, V(\bar{f})$, to be the number of the 3 -simplices of $B^{3}$ which are mapped onto 3-simplices of $X$. We define the volume of $f, V(f)$, to be

$$
V(f)=\min \{V(\bar{f})\}
$$

where $\bar{f}$ is an extension of $f$ with the properties mentioned earlier. We define the area of $f, A(f)$, to be the number of 2-simplices of $S^{2}$ which are mapped onto 2-simplices of $X$ by $f$.

If there is a group $G$ acting discretely and co-compactly on $X$ we say that $X$ is a 2-Dehn complex of $G$. In this case we define the second Dehn function of $X$, $\delta_{X}^{2}: \mathbb{N} \longrightarrow \mathbb{N}$, by

$$
\delta_{X}^{2}(n)=\max _{A(f) \leqslant n}\{V(f)\}
$$

where $f$ is a simplicial map $f: S^{2} \longrightarrow X$. 
Note that if $Y$ is another 2-Dehn complex of $G$ then one has $\delta_{Y}^{2}(n) \leqslant C \delta_{X}^{2}(C n)+C$ for some constant $C>0$.

We remark that there is a difference between $\delta_{X}^{2}$ and $\delta_{X}$, the Dehn function of $X$; there are only finitely many closed paths of length $n$ in $X$, modulo the action of $G$, while there are infinitely many inequivalent simplicial maps $f: S^{2} \longrightarrow X$ with $A(f) \leqslant n$, so a priori it is not even clear that $\delta_{X}^{2}(n)$ is finite for all $n \in \mathbb{N}$. The following proposition however insures that $\delta_{X}^{2}$ is well defined and in fact subrecursive. This again contrasts with the fact that there are groups $G$ for which $\delta_{X}$ is not bounded by any recursive function. The idea of the proof that follows is that 2-spheres of area $n$ correspond to 'Van Kampen diagrams' of area $n$ (by cutting the sphere along an edge one obtains a disk) and such Van Kampen diagrams can be listed.

Proposition 2.1. Let X be a 2-Dehn complex of a finitely presented group $G$. Then $\delta_{X}^{2}$ is bounded by a recursive function.

Proof. We consider first simplicial maps $f: S^{2} \longrightarrow X$ for which there is a simplicial subdivision of $S^{2}$ such that $f$ restricted in the interior of each 2-simplex of the subdivision is a homeomorphism. We call such maps non-singular. It is easy to see that, modulo the action of $G$, there is a finite number of such maps of area $n$. Indeed there is a finite number of inequivalent subdivisions of $S^{2}$ into $n$ 2-simplices.

For each such subdivision we pick a vertex * (base point) and we consider maps taking * to a fixed vertex, $e$, of $X$. Each such map $f$ induces a labelling of the simplices of the subdivision of $S^{2}$, namely each simplex $\sigma$ is labelled by the simplex $p(f(\sigma))$ where $p: X \longrightarrow X / G$ is the natural projection map. We denote the label of $\sigma$ by $l(\sigma)$. We call such labelled simplicial subdivisions of $S^{2}$ spherical diagrams. It is clear that there is a finite number of such diagrams of area $n$ and in fact it is easy to construct them all. Indeed we can construct all possible simplicial subdivisions of $S^{2}$ into $n$ simplices and consider all possible labellings of their simplices. A labelling corresponds to a spherical diagram if for all simplices $\sigma, \tau$ we have $l(\tau) \subset l(\sigma)$ when $\tau \subset \sigma$.

Moreover a map $f$ is completely determined by its corresponding based labelled diagram. Every simplicial map $f: S^{2} \longrightarrow X$ for which there is a simplicial subdivision of $S^{2}$ such that $f$ restricted in the interior of each 2-simplex of the subdivision is a homeomorphism is equivalent under the action of $G$ to a map $h: S^{2} \longrightarrow X$ described by a based spherical diagram (that is, $f=g h, g \in G$ ).

Let $f$ now be a map given by a spherical diagram $S$. We know that $f$ can be extended to $\bar{f}: B^{3} \longrightarrow X$ where $\left.\bar{f}\right|_{\partial B^{3}}=\left.f\right|_{\partial B^{3}}$. Indeed we can enumerate all simplicial subdivisions of $B^{3}$. For each one of them we see if there is a labelling such that the labelling of $\partial B^{3}$ is equal to $S$. Again we consider only labellings for which we have $l(\tau) \subset l(\sigma)$ when $\tau \subset \sigma$. Since we know that $f$ admits an extension $\bar{f}$ we will eventually find such a subdivision of $B^{3}$. The number of 3-simplices of this subdivision which are labelled by 3 -simplices of $X / G$ gives a bound for $\operatorname{Vol}(f)$. Since there is a finite number of inequivalent non-singular maps of area $n$ we can find a bound for the volume of all these maps.

We consider now singular simplicial maps $f: S^{2} \longrightarrow X$ of area $n$. Let $S$ be the simplicial subdivision of $S^{2}$ induced by $f$. We collapse successively the simplices of $S$ mapped to simplices of strictly lower dimension by $f$. $S$ is then transformed to a collection of spheres $S_{1}, \ldots, S_{r}$ joined by simple paths, or intersecting along simple 
ISODIAMETRIC AND ISOPERIMETRIC INEQUALITIES FOR COMPLEXES AND GROUPS 103

paths. $f$ induces a labelling to each of these spheres. Now the volume of $f$ is clearly bounded by the sum of the volumes of $\left.f\right|_{S_{i}}, i=1, \ldots, r$. As $r \leqslant n$ and area $\left(S_{i}\right) \leqslant n$, $i=1, \ldots, n$, our analysis of non-singular maps permits to find a bound of the volume of all simplicial maps $f: S^{2} \longrightarrow X$ of area $n$. In fact we gave an algorithm to find this bound which shows that $\delta_{X}^{2}$ is bounded by a recursive function.

Proposition 2.1 has been generalized recently to second order Dehn functions of finite derivation type monoids by Pride and Wang (see [14]).

It is known that there is a relationship between distortion of subgroups and isoperimetric inequalities. In particular one can construct examples of groups whose Dehn function grows faster than any recursive function by amalgamating groups along subgroups whose distortion function grows faster than any recursive function. There is an analogous relationship between area distortion of subgroups and second Dehn functions. We recall here the definition of area distortion (see [7, 9]).

DEFINITION 2.2. Let $H$ be a finitely presented subgroup of a finitely presented group $G$. Let $\mathscr{P}=\langle A \mid R\rangle, \mathscr{Q}=\langle B \mid T\rangle$ be finite presentations of $H$ and $G$ respectively. We choose 2 so that $A \subset B, R \subset T$. Let $w$ be a word on $A$ representing the identity in $H$. We denote by $\operatorname{area}_{\mathscr{P}}(w)$ the area of $w$ with respect to the presentation $\mathscr{P}$ and by $\operatorname{area}_{2}(w)$ the area of $w$ with respect to the presentation 2 . We define the area distortion function of $H$ in $G, f: \mathbb{N} \longrightarrow \mathbb{N}$, by

$$
f(n)=\max _{\operatorname{area}_{2}(w) \leqslant n} \operatorname{area}_{\mathscr{P}}(w)
$$

where $w$ runs over all trivial words on $A$.

Proposition 2.1 suggests then that the area distortion of subgroups is bounded by a recursive function. Indeed we have the following proposition.

Proposition 2.3. Let $G, H, \mathscr{P}, \mathscr{Q}, f$ be as in Definition 2.2. Then $f$ is a recursive function.

Proof. We will describe an algorithm which computes $f(n)$.

We first show how to compute a bound for $f(n)$. We consider the set of all words $w$ on $A$ with $\operatorname{area}_{2}(w) \leqslant n$. A minimal Van Kampen diagram $D$ of a word $w$ is topologically a union of disks $D_{1}, \ldots, D_{r}$ with simple paths joining them. Clearly $r \leqslant$ $n$. If $w_{i}$ is the boundary of $D_{i}$ we have length $\left(w_{i}\right) \leqslant M n$ where $M=\max \{\operatorname{length}(r)$ : $r \in T\}$. Therefore

$$
w_{i}=\prod_{j=1}^{k} u_{j} r_{j}^{ \pm 1} u_{j}^{-1}
$$

where $k \leqslant n$, length $\left(u_{j}\right) \leqslant(M+1) n, r_{j} \in T$ and the equality is in $F(B)$. It is clear that there is a finite number of products $\prod_{j=1}^{k} u_{j} r_{j}^{ \pm} u_{j}^{-1}$, with the above restrictions; we can therefore enumerate them and find all words on $A$ which could be boundaries of a diagram $D_{i}$ as above. All these words represent the identity in $G$; hence they are trivial in $H$ also. Therefore we can calculate their areas. Suppose the maximum area of all these is equal to $C$. Then clearly $f(n) \leqslant r C \leqslant n C$, that is, we have found a computable bound for $f(n)$. 
We used above the fact that if we know that a word $w$ is trivial in a group $H=$ $\langle A \mid R\rangle$ then we can calculate its area. This is done as follows. We first enumerate all products of the form $\prod_{j=1}^{k} u_{j} r_{j}^{ \pm 1} u_{j}^{-1}\left(u_{j} \in F(A), r_{j} \in R\right)$ until we find a product such that

$$
w=\prod_{j=1}^{k} u_{j} r_{j}^{ \pm 1} u_{j}^{-1} .
$$

Then we know that $A(w) \leqslant k$ and therefore we only need to check if $w$ is equal to an expression of the form $\prod_{j=1}^{m} u_{j} r_{j}^{ \pm} u_{j}^{-1}$ with $m<k$. There are infinitely many such expressions but it is enough to check only these expressions for which length $\left(u_{j}\right) \leqslant$ $k+$ length $(w)$ as the diameter of a Van Kampen diagram for $w$ of area $m$ is bounded by $m+$ length $(w)$ (see for example [11, Chapter 6]).

We now give an algorithm to compute $f(n)$, given the bound $f(n) \leqslant n C$ that we calculated above.

First we consider all ordered $k$-tuples (with repetition) $\left(r_{1}, \ldots, r_{k}\right)$, where the $r_{i}$ are relators of $\mathscr{Q}$ and $k \leqslant n$. To each such $k$-tuple, say $q$, we associate a product $\prod_{i=1}^{k} x_{q i} r_{i} x_{q i}^{-1}$, where the $x_{q i}$ are distinct variables. Clearly there are finitely many such products.

Similarly, we consider $k$-tuples of relators of $\mathscr{P}$ and products as before, where $k \leqslant n C$. Again, there are finitely many different products of relators.

We consider all systems of equations and inequations on $F(B)$ (the free group generated by $B$, the set of generators in 2$)$, obtained as follows. Each system has as the first equation $x=$ a product of conjugates of relators of $\mathscr{Q}$ as above, and for each product $p$ of conjugates of relators of $\mathscr{P}$ as above either the equation $x=p$ or the inequation $x \neq p$ is in the system.

We note that all variables appearing in different products are distinct, and $x$ is a variable that does not appear in any product.

We have a finite number of systems of equations and inequations as above, and each one of them can be algorithmically solved by a result of Makanin [17]. We note that such a solution determines $\operatorname{area}_{\mathscr{P}}(w)$ for a word $w$ with $\operatorname{area}_{2}(w) \leqslant n$. By solving all these systems, we can obtain the value of $f(n)$.

D. Cohen's result can be extended to higher dimensions.

THEOREM 2.4. Let $X$ be a locally finite 2-connected simplicial complex. There are constants $a, b$ such that if $f: S^{2} \longrightarrow X$ is a simplicial map from the 2-sphere to $X$ then

$$
\operatorname{vol}(f) \leqslant \operatorname{area}(f) a^{b^{\mathrm{diam}(f)}} .
$$

Moreover this 'double exponential' bound of volume in terms of area is 'best possible'.

Proof. We consider the 2-skeleton of $X, X^{(2)}$. If $v$ is a vertex in $X^{(2)}$ lying in the image of $f$, a set of generating elements for $\pi_{2}\left(X^{(2)}\right)$ is given by the set of spheres corresponding to boundaries of 3-simplices of $X$, joined to $v$ by paths. Let us denote this set by $A$. By the Hurewicz isomorphism theorem the set of boundaries of 3simplices of $X$ gives a set of generators also for $H_{2}\left(X^{(2)}\right)$. Let $c=[f]$ be the homology class of $f$ in $H_{2}(X)$. We have then

$$
c=k_{1} s_{1}+k_{2} s_{2}+\ldots+k_{n} s_{n}
$$

where $k_{i} \in \mathbb{N}, s_{i} \in A, i=1,2, \ldots, n$. 
By our hypothesis we know that there is a set of $s_{i}$ verifying (3) such that the distance of each $s_{i}$ from $v$ is less than $\operatorname{diam}(f)$. If we consider this set of $s_{i}$ we have

$$
n \leqslant b^{(\operatorname{diam}(f))}
$$

where $b$ is a constant depending only on $X$ (to be precise we take $b$ to be an integer bigger than the number of simplices adjacent to any given simplex of $X$ ).

$c$ is represented by a chain:

$$
c=m_{1} t_{1}+m_{2} t_{2}+\ldots+m_{r} t_{r}
$$

where $m_{i} \in \mathbb{N}(i=1, \ldots, r)$ and $t_{i}(i=1, \ldots, r)$ are 2-simplices of $X$. Clearly we can choose $m_{i}$ so that $\sum_{i=1}^{r}\left|m_{i}\right| \leqslant \operatorname{area}(f)$ for all $i$.

By substituting each $s_{i}$ in (3) by a sum of four 2-simplices and $c$ by the equation above, we reduce (3) to a linear system of at most $4 n$ equations with unknowns $k_{1}, \ldots, k_{n}$. We note that the coefficient of each $k_{i}$ in every equation of the system is $-1,0$ or 1 . Moreover it is different from 0 in at most $b$ equations.

Therefore if we take $n$ linearly independent equations of the linear system considered above, the determinant of the system of these $n$ equations will be bounded by $b^{n}$. Hence (3) admits a solution where $\left|k_{i}\right| \leqslant \operatorname{area}(f) b^{b^{\mathrm{diam}(f)}}$ for all $i$. By the Hurewicz isomorphism theorem equation (3) is valid in $\pi_{2}\left(X^{(2)}\right)$ as well which clearly implies the double exponential bound stated in the theorem.

We can see that this 'double exponential' bound is optimal in the same way as in Theorem 1.2. We construct a locally finite 2-connected complex and a sequence of balls with volume greater than the double exponential of their diameter. Our basic building blocks are homeomorphic to $S^{2} \times[0,1]$ with a simplicial subdivision so that area $\left(S^{2} \times 1\right)=2$ area $\left(S^{2} \times 0\right)$. Using these 'blocks' we construct a ball $B$ with boundary area and diameter $\sim n$ and volume $\sim 2^{n}$. We identify the boundary of this ball with the 'outer sphere' of our building block using a 'doubling map'. We repeat this $2^{n}$ times and finally we decrease the diameter of the singular ball obtained in the same way as in Theorem 1.2.

\section{References}

1. J. Alonso, W. Bogley, R. Burton, S. Pride and X. Wang, 'Second order Dehn functions of groups', Quart. J. Math. Oxford Ser. (2) 49 (1998) 1-30.

2. J. M. Alonso, S. J. Pride and X. WANG, 'Higher dimensional isoperimetric (or Dehn) functions of groups', J. Group Theory 2 (1999) 81-112.

3. D. COHEN, 'Isodiametric and isoperimetric inequalities for group presentations', Internat. J. Algebra Comput. 1 (1991) 321-327.

4. D. EPSTEIN, Word processing in groups (Jones and Bartlett, Boston, 1992).

5. S. Frankel and M. Katz, 'The Morse landscape of a Riemannian disk', Ann. Inst. Fourier (Grenoble) 43 (1993) 503-507.

6. S. M. Gersten, 'The double exponential theorem for isodiametric and isoperimetric functions', Internat. J. Algebra Comput. 1 (1991) 315-320.

7. S. M. Gersten, 'Preservation and distortion of area in finitely presented groups', Geom. Funct. Anal. 6 (1996) 301-345.

8. M. Gromov, 'Hyperbolic groups', Essays in group theory, Mathematical Sciences Research Institute Publications (ed. S. M. Gersten, Springer, 1987).

9. M. Gromov, 'Asymptotic invariants of infinite groups', Geometric group theory-Vol. 2, London Mathematical Society Lecture Note Series 181 (ed. G. Niblo and M. Roller, Cambridge University Press, 1993).

10. A. Hatcher and K. Vogtmann, 'Isoperimetric inequalities for automorphism groups of free groups', Pacific J. Math. 173 (1996) 425-441.

11. R. LYNDON and P. SChUPP, Combinatorial group theory (Springer, 1977).

12. K. Madlener and F. Otто, 'Pseudo-natural algorithms for the word problem for finitely presented monoids and groups', J. Symbolic Comput. 1 (1985) 383-418.

13. P. Papasoglu, 'Quasi-flats in semi-hyperbolic groups', Proc. Amer. Math. Soc. 126 (1998) 1267-1273. 
106 ISODIAMETRIC AND ISOPERIMETRIC INEQUALITIES FOR COMPLEXES AND GROUPS

14. S. J. Pride and X. WANG, 'Second order Dehn functions of groups and monoids', preprint.

15. E. H. SPANIER, Algebraic topology (Springer, 1966).

16. $\mathrm{X}$. WANG, 'Second order Dehn functions of finitely presented groups and monoids', PhD Thesis, Glasgow, 1996.

17. G. S. Makanin, 'Decidability of the universal theory and positive theory of free groups', Izv. Akad. Nauk SSSR Ser. Mat. 48 735-749 (Russian); Math. USSR Izv. $2575-88$ (English).

Département de Mathématiques

Université de Paris-sud

BAT 425

91405 Orsay

France

papazog@topo.math.u-psud.fr 